\title{
SIMULATING FLIGHT CONDITION OF HFSSB ON GROUND
}

\begin{tabular}{|l|l|l|l|}
\hline Sampada Kubsad, & Suvarna P, & Anusha A. Shetty, & Yogesh Tiwari, \\
Assistant Professor & Student & Student & Student \\
$\begin{array}{l}\text { Dept of ECE, Atria Institute } \\
\text { of Technology }\end{array}$ & Dept of ECE, Atria Institute of & Dept of ECE, Atria & Dept of ECE, Atria \\
sampada.kubsad@gmail.com & $\begin{array}{l}\text { Technology } \\
\text { suvarnanaidu1994@gmail.com }\end{array}$ & $\begin{array}{l}\text { Institute of Technology } \\
\text { anushetty22@ gmail.com }\end{array}$ & $\begin{array}{l}\text { Institute of Technology } \\
\text { yogesh.mar05@ gmail.com }\end{array}$ \\
\hline
\end{tabular}

\section{ABSTRACT:}

HF (high frequency) system is used for long distance communication in aircrafts from air to ground or ground to ground. Since it is not line of sight communication it is difficult to get reception in closed areas like hangars. If the communication system is found unserviceable during flights it is difficult to determine whether the problem lies in the LRU (line replaceable unit) or the loom (wiring). If the wiring is found proper it is still difficult to recognize and replace faulty LRU. To overcome these problems and to check the functionality we simulate flight condition on ground using test kit.

KEYWORDS: HFSSB, Communication, LRUs, PTT

\section{INTRODUCTION}

Communication systems play an important role in avionics. The various kinds of communication systems used are UHF, VHF, HF, ADF etc. HF systems are an integral part of the communication system and are used for long range communication. Testing HF system in aircraft has always been a tedious task. Here we present an opportunity to test the system in an easy manner.

\subsection{INTRODUCTION TO HFSSB}

HF communication systems are long-distance communication systems, many airlines that employ HF communication systems do so because these systems provide

an extended range of communication between aircraft and from aircraft to ground stations. The highfrequency communication systems operate in the frequency range of 2.0 to $30 \mathrm{MHZ}$, the $\mathrm{HF}$ range is actually a middle-frequency range, in as much as it starts above the standard broadcast band, which ends at approximately $1700 \mathrm{KHZ}$. The HF system on an airplane is used to provide two way voice communication.

The HF radio control panel is located where it is easily accessible to pilot or co-pilot

The HF system consists of HF receiver-transmitter, HF controller unit, antenna coupler system, and antenna. The different modes in HF systems, single sideband(SSB), upper sideband(USB), lower sideband(LSB), full carrier amplitude modulation(AM), continuous wave(CM), data modes. The mode used here is HF system with single sideband [1]

\subsection{BRIEF DISCRIPTION ABOUT} COMMUNICATION SYSTEM

Single sideband: Single sideband modulation (SSB) or Single-Sideband Suppressed-Carrier (SSB-SC) is a refinement of amplitude modulation which uses transmitter power and bandwidth more efficiently. Amplitude modulation produces an output signal that has twice the bandwidth of the original baseband signal. Single-sideband modulation avoids this bandwidth doubling, and the power wasted on a carrier, at the cost of increased device complexity and more difficult tuning at the receiver.

Simplex communication: The transmission and reception of the communication signal on the same frequency is known as simplex communication.

Semi - duplex communication: Transmission on one frequency and receiving on other frequency of communication signals is known as semi - duplex communication.[2] 


\section{RELATED WORK}

HAL (HINDUSTAN AERONAUTICALS LIMITED) maintains a multitude of combat helicopters such as ALH. The communication system is based on VHF communication. Also $\mathrm{HF}$ communication is used as a backup option for $\mathrm{VHF}$ communication system. These communication systems were based on INTERCOM system which connected these systems to the station box. So there is no system which can test the functionality of the HF system. So any snag related to the HF communication was rectified by replacing the individual LRUs. These LRUs were replaced on ground and trial and error method was used to determine the faulty component. The fault can be with the loom or the LRUs. So its not possible to check the looms in the aircraft so it usually took 3 flights to determine whether the fault lied in the loom or the LRUs.[6] Also the individual LRUs had to be taken out from the aircraft to test them in the lab. To just test the system's working, the aircraft had to be towed out of the hangar in open space where the whole aircraft was powered up to test the communication system.

\section{PROPOSED WORK}

To solve the above stated problem, we have implemented a Test kit [fig.1]. Test kit is an equipment designed to simulate and check the functionality of HF system on ground independently by bypassing power and intercom system by implementing its functionalities.

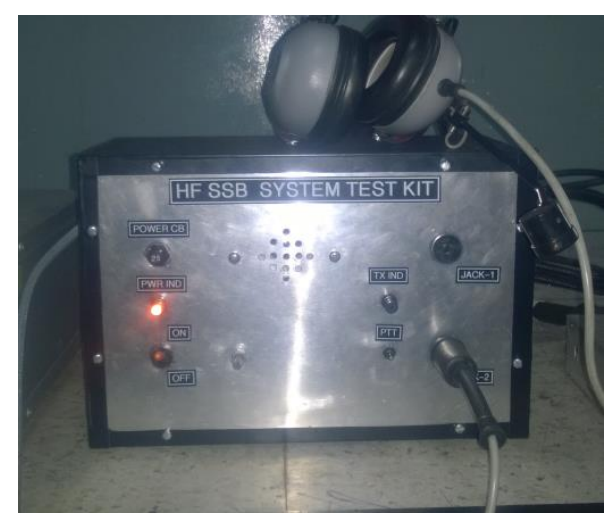

Fig1: Test kit
Test kit is used to bypass the intercom system and we provide some basic functionalities of the intercom system[8] in the test kit. It also displays the signal strength of transmitter and receiver separately. With this kit it's easy to identify the faulty LRU of HF system and to reconfirm the problem of faulty LRU before sending for repair. The inputs to the system are from the control and display unit and the audio signals from the microphone. The output of the system is checked through headset. Test kit consists of power supply distribution system through circuit breaker and fuse box, control switches for on/off, volume control and PTT, RF amplifier to amplify the microphone and audio levels[3].

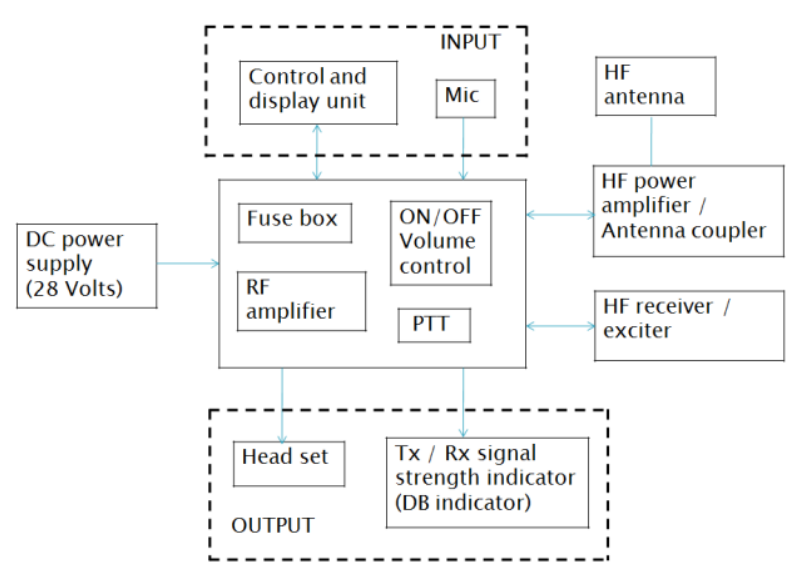

Fig. 2 block diagram of HFSSB test kit

\section{HARDWARE DESCRIPTION}

This system consists of 4 LRUs. Control Display Unit, Receiver Exciter, Power Amplifier / Antenna Coupler, Antenna.

4.1 Receiver Exciter: The exciter is a transmitter that produces a very low power signal, which is fed to the power amplifier. While receiving, the signal from the antenna passes through the antenna coupler, but bypasses the amplifier section [fig 3].This received signal will be demodulated in the Receiver / Exciter and fed to the headset as an audio output. Receiver Exciter unit is installed on the hinged platform on RH side in avionics bay. 


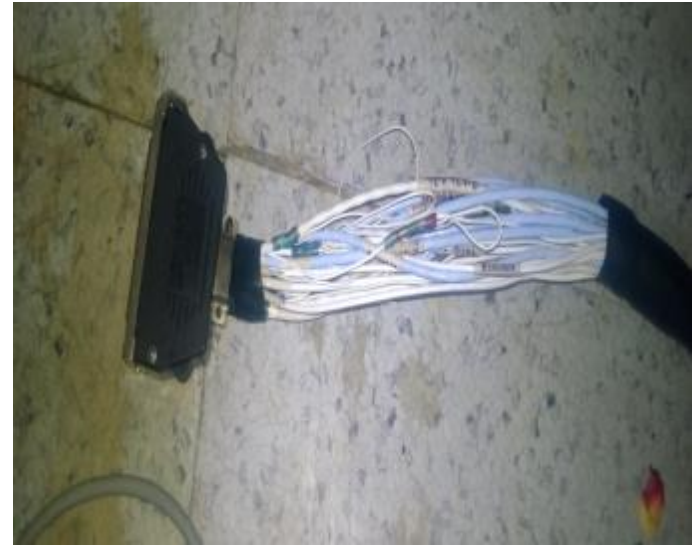

Fig. 3 connector

4.2 Power Amplifier / Antenna Coupler: Exciter Power amplifier / Antenna coupler is mounted near the antenna to optimize transmission efficiency. The power amplifier amplifies the signal from the receiver / exciter into a 150 watts Peak Envelop Power (PEP) transmitted signal in Single Side Band operation [4] and 37.5 watts carrier power and 150 watts PEP in AM operation. The antenna coupler changes its electrical impedance to tune the antenna to each frequency, making antenna appear to the transmitted signal as though it were the optimum physical length. While receiving, the signal from the antenna passes through the antenna coupler, but bypasses the amplifier section. The Power Amplifier / Antenna Coupler are installed on top rack of $\mathrm{RH}$ side in avionics bay.

4.3 Control Display Unit: It facilitates to select different modes and the various frequencies for communication[5] with the ground stations and the other aircrafts. It is located in the centre console of ALH.

4.4 Antenna: It is a grounded tramline (transmission line), often referred to as "towel bar" antennas[9] because their shape resembles a towel bar. These are best suited for helicopter applications. It is attached parallel to the tail boom. It consists of a lead-in mast, two support masts and a grounded mast. The masts are designed to provide high efficiency; lead-in and grounding connections. The antenna is installed on the RH side of the tail loom.

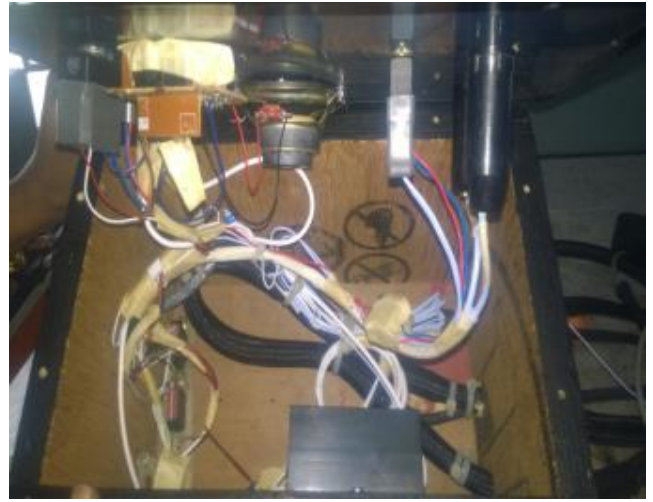

Fig. 4 looming inside test kit

The test kit here bypasses the intercom system and therefore employs its functionality of PTT (Push to talk) switch, mic and audio. The test kit comprises of a 25Amps circuit breaker, power on switch and an indicator, volume control knob, PTT switch, Tx indicator, and audio jacks. The test kit is given a power supply of $28 \mathrm{~V}$ DC through a fuse box which prevents the LRUs from high voltage. Most of the looms are shielded which reduce the effect of interference in the system. These shields are grounded by using an earth module which in turn is connected to the body of the test kit. The headsets are connected to the audio jacks for clear reception or transmission.[6]

\section{RESULT}

To test the system's performance in a real aircraft a CHEETAH (fig. 5) helicopter was used.

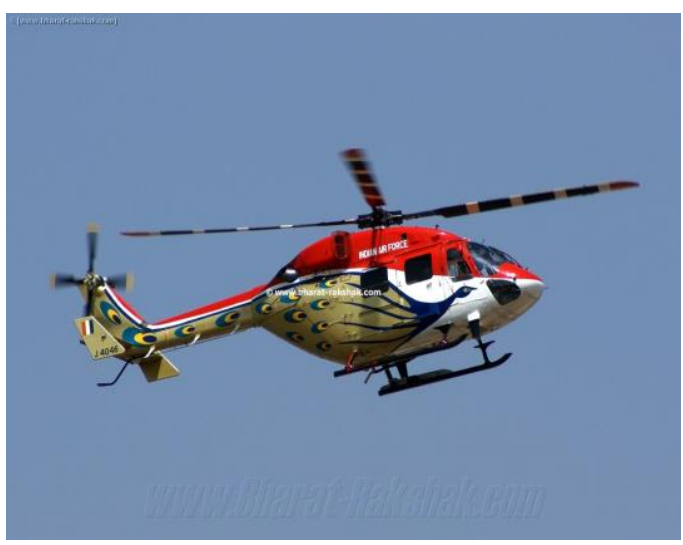

Fig. 5 Cheetah chopper 
We connected this test kit to the different LRUs. The system was powered up by a $28 \mathrm{~V}$ DC supply. The test kit power button was turned on which was indicated by the power indicator. Then the PTT switch was released to use the system in the reception mode. The headphone was inserted on the audio jacks. We were able to receive the communication by tuning the control unit at $1535 \mathrm{MHz}$ frequency. This confirmed that the communication system was proper and could be used in flights.

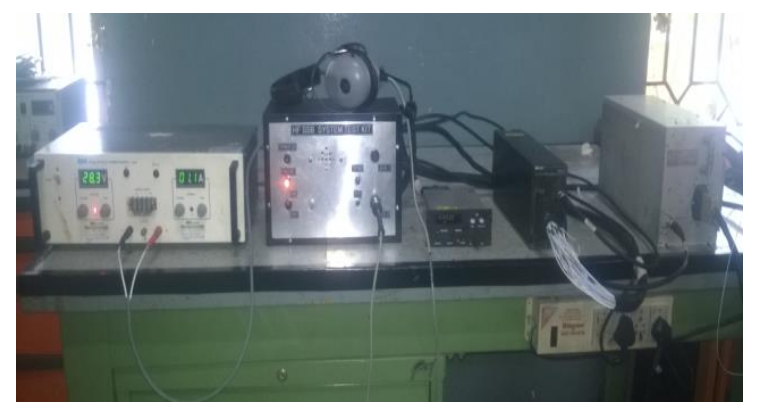

Fig. 6 entire test setup

\section{APPLICATION}

It is possible to check the proper reception or transmission inside the hangar, .It reduces man power and with limited use of resources, it is possible to check all the LRU's, Resources means it may be fuel or man and mechanical power to push the helicopters outside, We determine whether the transmission and reception of signals are possible without any noise with a zero flight.

\section{ADVANTAGES}

It enables us to determine the source of fault. On an average it takes about 3 flights to determine the cause of the snag in the communication system. This system can enable us to reduce it to 1 flight. Also the there is no need for the aircraft to be taken outside for testing as this test kit can be setup on a table. So it enables us to prevent the whole aircraft to be powered up which consumes enormous amount of power as we only power up the test kit which takes up $28 \mathrm{~V} \mathrm{DC}$.

\section{FUTURE SCOPE:}

The test kit is not equipped to determine the exact LRUs which cause the snag in the communication system. Also this system can be equipped with a DB meter to specify the amount of power reaching each system. This may further help us in determining the amount of attenuation occurring in the system. In future such problems can be overcome by further research.

\section{CONCLUSION}

This state of the art test kit has enabled us to test the HF communication system like never before. It has allowed us to create a standalone system for testing the system. Also we have radically devised this kit to work on a table which makes it extremely easy to operate. This has been able to cut down the manpower and the time needed to rectify the snag in the communication system. So what took 3-4 days of hard work can now be reduced to mere 10 minutes of work. This test kit also reduces the cost inflicted every time an aircraft was towed out to fly to test the communication system from 3-4 lakhs to virtually zero cost.

\section{REFERENCES}

[1]Frederick H. Raab, Robert Caverly, Richard Campbell, Murat Eron, James B. Hecht, Arturo Mediano, Daniel P. Myer, and John L. B. Walker," HF, VHF, and UHF Systems and Technology" ieee transactions on microwave theory and techniques, vol.50, no.3, march 2002.

[2] Modern HF systems for National Meteorological Telecommunication Networks, coordination meeting on the GTS, strategy in Africa, geneva, 30 OCT. - 3 NOVEMBER 2000.

[3]sgc - "HFSSB communications guide book".

[4] Mike Tooley, David Wyatt-"Aircraft Communications and Navigation Systems: Principles, Maintenance and Operation". [5] Dale Stacey "Aeronautical Radio Communication Systems and Networks". [6] Federal Aviation Administration"International Journal of Applied Aviation Studies", Volume 7, Number 2, $2007 . \quad$ [7] Mike Tooley, David Wyatt-"Aircraft Electrical and Electronic SystemsPrinciples, Operation and Maintenance”. 
[8] Padfield,G.D.,1996, Helicopter flight dynamics: the theory and application of flying qualities and simulation modelling. AIAA Education Series, Washington,DC. [9]A.Tzikas,D Chrissoulidis, E.Kriezis. Journal: IEEE Transactions on Antennas and Propagation - IEEE TRANS ANTENNAS PROPAGAT , vol. 34, no. 8, pp. 1046-1052, 1986. 